\title{
La universidad latinoamericana frente a la globalización. Continuidades e inflexiones de un mismo proceso (1968 y 2011)*
}

\begin{abstract}
Resumen
El objetivo de este artículo es analizar las dinámicas de la globalización y de la tecnocracia en escenarios como la educación superior. El texto hace parte de un proyecto investigativo que busca preguntarse por dos dinámicas sociales: la primera presente en el año de 1968 y la segunda en el año 2011. La propuesta va dirigida no solo a explicar las tensiones movilizatorias en medio de un panorama globalizado, sino identificar la dinámica glocal como cualidad performadora de las sociabilidades desde la segunda mitad del siglo XX hasta la actualidad.
\end{abstract}

Palabras Clave: globalización, movilización, tecnocracia.

Referencia para citar este artículo: ACEVEDO TARAZONA, Álvaro y CORREA LUGOS, Andrés (2017). "La universidad latinoamericana frente a la globalización. Continuidades e inflexiones de un mismo proceso: 1968 y 2011”. En Anuario de Historia Regional y de las Fronteras. 22 (2). pp. 121-139.

\begin{abstract}
Álvaro Acevedo Tarazona: Posdoctor en Ciencias de la Educación de la Universidad Pedagógica y Tecnológica de Colombia. Doctor en Historia de la Universidad de Huelva (España). Magíster en Historia e Historiador de la Universidad Industrial de Santander, Magíster en Historia de América Latina de la Universidad Pablo de Olavide (España). Formación Especializada Docencia en Historia y Cultura de América Latina de la Universidad Pablo de Olavide (España). Especialista en Filosofía de la Universidad de Antioquia. Profesor titular de la Universidad Industrial de Santander, Colombia. Correo electrónico: tarazona20@gmail.com. Código ORCID: 0000-0002-3563-9213.
\end{abstract}

Andrés Correa Lugos: Historiador y Archivista de la Universidad Industrial de Santander. Miembro activo del grupo de investigación "Politicas, sociabilidades y representaciones histórico-educativas", Universidad Industrial de Santander, Colombia. Correo electrónico: andrescorrealugos@outlook.com. Código ORCID: 0000-0002-6477-8001.

\footnotetext{
* Este artículo es parte del proyecto de investigación El ocaso de la utopía antisistémica: el conflicto, la universidad y la apuesta por la paz en Colombia 1968-1998 financiado por Colciencias y la Universidad Industrial de Santander, código: 8262.
} 


\title{
The Latin American University Against Globalization. Continuities and Inflections of the Same Process: (1968 and 2011)
}

\begin{abstract}
The aim of this article is to analyze the dynamics of globalization and technocracy in scenarios such as the higher education. The text is part of a research project that pursues to ask after two social dynamics: the first in the year 1968 and the second in 2011. The proposal is addressed not only to explain the mobilizing tensions in the middle of a Globalized panorama, but to identify the global dynamics as the constructive quality of sociabilities from the second half of the twentieth century to the present.
\end{abstract}

Keywords: Globalization, Mobilization, Technocracy.

\section{A universidade latinoamericana contra a globalização. Continuidades e inflexões de um processo: (1968 e 2011)}

\begin{abstract}
Resumo
O objetivo deste trabalho é analisar as dinâmicas da globalização e da tecnocracia em âmbitos como o ensino superior. $O$ texto faz parte de um projeto de pesquisa que almeja perguntar por duas dinâmicas sociais: a primeira, em 1968, e a segunda, em 2011. A proposta não aborda somente a explicação das tensões mobilizadoras em meio a um panorama globalizado, mas também a identificação da dinâmica glocal como qualidade geradora de sociabilidades desde a segunda metade do século XX até o presente.
\end{abstract}

Palavras-chave: Globalização, mobilização, tecnocracia. 


\section{Introducción}

El presente artículo pretende acercar al lector al conocimiento de las dinámicas globalizadas y tecnócratas en el Latinoamérica, siendo más específicos en países como Chile, Colombia y México. La elección de estas realidades locales se debe a que comparten una linealidad histórica, pero difieren de manera considerable en la actuación y/o respuesta ante los postulados globalizados y tecnócratas. A pesar de las diferencias de cada localidad, el texto trata de dilucidar los puntos de encuentro y desencuentro a la par que se va construyendo una lógica globalizada que tiene como finalidad la capitalización del sí-mismo, basándose en términos generales, en la naturaleza de la oferta universitaria de la región.

En el desarrollo del texto se analiza el proyecto globalizador y las repercusiones que este ha establecido no sólo en la forma como los actores sociales se desenvuelven en la vida cotidiana, sino en la forma como protestan frente a los postulados globalizados. La metodología de la triangulación permite acercar los planteamientos teóricos a las realidades latinoamericanas. En razón de este propósito, muestra acontecimientos como la revolución del 68 no solo como una fractura cultural sino como todo un evento político que reconfigura la participación ciudadana de la juventud; a la par, se pregutna por las movilizaciones del año 2011 como una coyuntura planetaria dirigida desde pretensiones locales. El artículo hace énfasis en el concepto de glocalización; para ello, dispone de un primer apartado de acercamiento teórico en el que busca acercar el conocimiento del concepto de globalización con nuevas alternativas analíticas de la globalidad.

Una vez diferenciados los conceptos se busca las especificidades de la relación entre lo global y lo glocal en un marco de problemas compartidos en América Latina. Para ello se muestra la necesidad de un proyecto globalizador como una lógica dominante que propone superar los baches de guerras mundiales, conflictos geopolíticos y las brechas espacio temporales existentes. Este recorrido llega hasta el año de 1968 como un punto de inflexión globalizado, pero también como un proceso de emancipación y revolución que dará pie no solo a una nueva forma de protesta, sino a una nueva forma de consumo y capitalización del mundo. Este proceso algunas veces imperceptible pero realizado a gran escala, sumerge al planeta entero en una lógica globalizada en la cual la universidad es también parte de este proceso, bajo criterios de eficiencia, eficacia y calidad. De esta manera se implementa una educación no al servicio de la sociedad y dignificación del ser humano, sino como alternativa y complemento a nuevas tipologías laborales que genera recursos.

En esta dinámica globalizada aparece la última inflexión que desarrolla el texto: es a partir del año 2011 que las personas comparten una serie de pulsiones y molestias por el ritmo acelerado y el continuo endeudamiento del futuro (con créditos educativos, hipotecas, etc.). Inicia así una serie de manifestaciones locales que son mediatizadas por las redes sociales. Esta nueva forma de movilización no es antiglobalizadora, pero si critica el rumbo que está tomando al incrementar de manera considerable la desigualdad social. 


\section{Acercamiento teórico}

Construir un relato histórico de la globalización constituye un reto para el oficio del historiador porque necesita de una mirada pluridimensional de los acontecimientos, las transiciones $\mathrm{y}$, sobre todo, del reconocimiento de las etapas de corta, mediana y larga duración. Además de estos conocimientos propios de la experiencia, es importante recurrir a un uso adecuado de los conceptos. En razón de ello, se desarrolla como primera medida para la construcción del texto, unos apartados que sintetizan el trabajo realizado por los autores en temas como: globalización, glocalización, tecnocracia y movimientos sociales.

La globalización es un concepto bastante denso, está presente a diario en las sociabilidades del siglo XXI: globalización en los medios de comunicación, en la educación, en la política, en la economía, en los deseos, en las pasiones y hasta en las pulsiones. Es tarea entonces del investigador social tomar con rigurosidad el concepto. Autores como Immanuel Wallerstein empalman la globalización con la aparición del sistema-mundo y la apertura del mundo eurocéntrico a nuevos encuentros con el otro ${ }^{1}$. Si bien este proceso es el dinamizador de lo que se conoce como la modernidad y da pie al afianzamiento del liberalismo y el capitalismo, solo sería casi cuatro siglos después cuando la globalización como concepto aparece.

La constitución de un concepto implica que exista un acontecimiento, una situación totalmente novedosa que induzca a la producción de un neologismo; debe ser un proceso tan fuerte que reconfigure el presente y habilite un futuro impensable sin él; debe articular el pasado que lo precede para explicarlo ${ }^{2}$. ¿Dónde encontrar un fenómeno de tal magnitud? La globalización inicia con la mundialización de la técnica, la tecnología y la ciencia a la par que se acrecienta el miedo a la violencia y a la catástrofe demográfica que deja un mundo que no solo comparte sus bienes sino también sus males. La globalización como concepto hace su aparición en el año de 1958 en la columna globalised quote de la revista the economist ${ }^{3}$, en plena posguerra, bajo la coyuntura de la necesidad de fundar una Comunidad Económica Europea mediante la cual se busque dinamizar procesos transculturales, transeconómicos y geopolíticos, al mismo tiempo que se potencializa un capitalismo industrial y un consumo elevado a nuevos targets poblaciones como es la "juventud".

La globalización aparece como un proceso cultural, económico y político referido a una serie de complejas problemáticas derivadas de los conflictos mundiales. Ante este hecho, se intenta retomar el discurso moderno del progreso y el liberalismo y potencializarlo con tecnologías de la información y la comunicación, la capitalización y las ganancias a través de nuevos espacios que van más allá de la fábrica y consoliden un mercado cultural y del entretenimiento; paralelo a ello se venden experiencias y sensaciones fugaces y obsolescentes. El resultado es asombroso, para 1968 el mundo

\footnotetext{
${ }^{1}$ Wallerstein, Immanuel. El moderno sistema mundial. Vol. III (Buenos Aires: Siglo XI editores, 1979).

${ }^{2}$ Zizek, Slavoj. Acontecimiento (Madrid: Sexto Piso, 2014), p. 181

${ }^{3}$ Walter, Malcolm. Globalization (Londres: Routledge, 1995), p. 8.
} 
se encuentra metido en una nueva lógica de consumo, la cual deriva en revoluciones culturales por todo el planeta; millones de jóvenes están en las calles exigiendo libertades y mostrando el desacuerdo ante un oficialismo sistémico que los ahoga. Por su parte, el sistema prepara intervenciones en todo el mundo para contener las euforias de rebeldía: el consumo puede seguir pero la toma de la libertad no. Eventualidades como la matanza en la Plaza de las Tres Culturas, en México, ad portas de unos Juegos Olímpicos, son evidencia que la globalización no solo trae consigo una liberación y un consumo desbocado sino que tiene métodos para crear cortinas de humo.

Ahora bien, El impulso de la globalización reconfigura los territorios, a la vez que complementa la manera como los actores sociales enfrentan nuevas territorialidades. La emergencia de coyunturas muy específicas, pero ahora visibilizadas por los medios de comunicación hace que el impacto de la globalización sea multidireccional. Desde esta lógica se empiezan a construir muchos discursos, en su mayoría reaccionarios, que buscan influenciar de manera positiva en los problemas locales, nacionales e internacionales. La conceptualización de esta nueva lógica lleva entonces a delimitar la noción de glocalización: lo glocal es la conjunción entre lo local y lo global. Este término es acuñado por el sociólogo Rolan Robertson en el texto glocalization: timeespace and homogeneity-heterogeneity; el término proviene del concepto japonés dochakuka cuya traducción es "el que vive en su propia tierra"4. Robertson explica que sólo cuando existe una suficiente conciencia de la importancia de las coyunturas locales es posible trascender a la construcción de una problemática en sincronía con el mundo.

La preocupación por el mundo empieza entonces a ser una aclamación visible en las generaciones intranquilas por la influencia que hacen las políticas trasnacionales en gobiernos nacionales tradicionalistas que no alcanzan ni siquiera a tener un impacto regional. En países del primer mundo se traduce en una movilización contra el imperialismo en otras regiones, el rechazo a las pruebas nucleares y el reclamo continuo a los inclusión y derechos de mujeres y minorías. En el segundo mundo se evidencia un rechazo al régimen comunista que limita una apertura económica y cultural con el resto del planeta. En el tercer mundo se produce una corriente antisistémica en contra del neocolonialismo y de las recursivas formas de dominación orientadas por organizaciones trasnacionales, como el Banco Mundial y el Fondo Monetario Internacional, para frenar las incursiones comunistas en dichas regiones. El teórico social Herbert Marcuse documenta la situación planetaria en dicha contemporaneidad:

En los países latinoamericanos que se encuentran bajo dictaduras fascistas o militares se lleva a cabo una cruel persecución. La tortura se ha convertido en un recurso normal de los "interrogatorios" de todo el mundo. Ahí donde la resistencia de los pobres ha sido vencida, los estudiantes encabezan la lucha contra la soldadesca y la policía; se asesina, se arrojan gases y se bombardea a los estudiantes, y se les apresa por centenares. Los Juegos Olímpicos fueron inaugurados con la persecución y muerte a tiros de trescientos de ellos en las

\footnotetext{
${ }^{4}$ Robertson, Roland. Glocalización: tiempo-espacio y homogeneidad-heterogeneidad (Madrid: Trotta, 2003), pp. 25-44.
} 
calles de ciudad de México. En los Estados Unidos, los estudiantes se mantienen a la vanguardia de la protesta radical: las matanzas en las universidades de Jackson State y Kent son prueba de su papel histórico [...] El capitalismo se organiza para enfrentarse a la amenaza de una revolución que sería la más radical de todas las revoluciones históricas, la primera verdaderamente mundial e histórica ${ }^{5}$.

Desde la perspectiva histórica y sociología de Marcuse, una compartida por pensadores de izquierda la época, los jóvenes y en especial los universitarios son la esperanza revolucionaria global en la historia de la sociedad. Las movilizaciones protagonizadas en las décadas de 1960 y 1970 llevan a pensar que tinen la suficiente fuerza de convocatoria y los ideales para lograr un cambio. Gran parte del desarrollo movilizatorio de los jóvenes se debe a que tienen un espacio autónomo en el cual dan vía libre a la construcción de un discurso y un pensamiento alternativo. Ese espacio es la universidad. El sistema, consciente de dicho escenario, inicia una serie de maniobras para obstruir el desenvolvimiento revolucionario y movilizatorio.

La estrategia para obstruir las dinámicas revolucionarias es mediante la consolidación de un sistema que ofrezca el beneficio moderno del progreso y no caiga en procesos políticos demagógicos y ultranacionalistas. Es en la posguerra cuando se busca la concentración del poder en el desarrollismo técnico y tecnológico; la ventaja que tiene este sistema para aplacar las movilizaciones es que anula la decisión y la voluntad, pues el credo tecnocrático sólo concibe la verdad objetiva de la ciencia ${ }^{6}$, no da pie a reinterpretaciones y mucho menos a revoluciones que no estén dirigidas a un cambio de paradigma. La tecnocracia surge como una alternativa para las relaciones de poder entre quien es dueño de los medios de producción y quien vende su fuerza de trabajo; es el primer paso hacia la capitalización o gerenciamiento del sí-mismo, o como dice Alain Touraine: distienden el equipo y el servicio ${ }^{7}$.

Existen diversos modelos tecnócratas ofertados según la realidad local, pero hay unos pilares fundamentales que deben ser tenidos en cuenta. En primer lugar, existe un cambio radical en la forma de considerar al estado y a la sociedad, pues estos son concebidos como sistemas. Al ubicar la noción del sistema, en segundo lugar, hay entonces un objetivo implícito: que los procesos de intervención social deben estar orientados por una razón técnica y ser cuantificados en términos instrumentales. En tercer lugar, el conocimiento no está sectorizado y puede ser aplicado a cualquier dimensión de la vida social, en otras palabras, abre la puerta a que prácticas técnicas, secuenciales e instrumentales sean aplicadas a políticas públicas en una ingeniería social. Por último, la tecnocracia predica que para cada problema siempre existe una solución óptima; la optimización no se cualifica sino que se cuantifica con factores como eficiencia y eficacia ${ }^{8}$.

\footnotetext{
${ }^{5}$ Marcuse, Herbert. Contrarrevolución y revuelta (México D.F: Ed. Joaquín Mortiz, 1973), pp. 11-12.

${ }^{6}$ Mayol, Alberto. La tecnocracia: el falso profeta de la modernidad, en Revista de Sociología, núm. 17, Santiago de Chile: Universidad de Chile, 2003, pp. 95-123.

${ }^{7}$ Touraine, Alain. La sociedad postindustrial (Barcelona: Ariel, 1973).

${ }^{8}$ Mayol, Alberto, Op Cit.
} 
Los postulados tecnócratas son potencializados por una era globalizada que se abre en todo el planeta. El desarrollismo encabeza las grandes reformas y cambios en distintas regiones, a la par que organizaciones transnacionales como el Banco Mundial y el Fondo Monetario Internacional giran millones de dólares a países, en especial del tercer mundo, para desarrollar los proyectos. Gran parte de dichos proyectos de desarrollo van encaminados a sectores productivos de la sociedad, los cuales necesitan primeramente de la capacitación y profesionalización de la mano de obra. Es por esta razón que las universidades se convierten en grandes polos e intereses del desarrollismo tecnócrata y globalizado. Con lo que no cuenta es que es en las mismas universidades donde se van a levantar todo tipo de movilizaciones en contra de tales pretensiones.

\section{Antecedentes históricos de la globalización tecnócrata}

Es al inicio del siglo XX cuando se hace necesaria una ideología que reemplace el ocaso ideológico decimonónico. Lo que no es posible desechar es la idea de progreso, pues es la utopía del progreso la que permite a la humanidad organizarse, resistir y producir en torno a un futuro mejor. La tecnocracia llega entonces para darle un segundo momento a los postulados filosóficos del progreso y hacerlo práctico. En los primeros veinte años del siglo XX se produce una aceleración del conocimiento sin precedentes, con avances científicos como la electricidad, los motores de combustión interna, los avances médicos en torno a las proteínas y las vitaminas, las primeras vacunas y el crecimiento progresivo de las ciudades en todo el mundo 9 . Para la década de los treinta la tecnocracia sobrevive a una Guerra Mundial y a la primera crisis económica mundial (1929). La expansión de campos técnicos de la producción tanto físicos como químicos, principalmente, conduce a que sean los científicos e ingenieros de estas especialidades los llamados a diseñar y ejecutar las políticas gubernamentales de la sociedad industrial en su conjunto. Un ejemplo de esto es el ascenso político, económico y científico de personajes como Robert McNamara. Después de una carrera exitosa dirigiendo la Ford Motor Company, este se convierte en secretario de defensa norteamericano, en plena Guerra de Vietnam, y luego es presidente del Banco Mundial hasta $1981^{10}$.

La administración de este proceso tecnócrata necesita de un impulso globalizado, y este tiene su causa fundamental después de la Segunda Guerra Mundial (1939-1945). Esta guerra representa para el mundo entero no solo la capacitación y puesta en práctica de tecnologías de punta -consideradas tan solo años atrás como meros prototipossino la pérdida de una generación completa de jóvenes que va a morir en territorios desconocidos. También representa el posicionamiento de dos potencias políticas, económicas e ideológicas que comparten la carrera tecnológica. El mundo deja de ser visto como un conjunto y ahora es pensado en dos bloques con la suficiente avaricia, poder y dinero para conquistar no solo la otra parte del mundo que no poseen sino el universo mismo (o eso es lo que los delirios de cada potencia, en plena Guerra Fría, hacen creer).

\footnotetext{
${ }^{9}$ Barraclough, Geoffrey. Introducción a la historia contemporanea (Madrid: Gredos, 1964), p. 52.

${ }^{10}$ George, Susan. Otro mundo es posible si... (Barcelona: Icaria , 2004), p. 95.
} 
La historia permite ver en este escenario temporal específico a dos protagonistas antagónicos (la Unión de Repúblicas Socialistas Soviéticas y Estados Unidos), una atribulada Europa y unos extras de reparto con apariciones ocasionales (África, Asia y América Latina). En el caso de América Latina el panorama es bastante complejo, tal vez menos bipolar como en otras latitudes del mundo pero no menos conflictivo. En el mismo año que se crea la Organización de Estados Americanos, 1948, en Colombia ocurre el asesinato del líder político Jorge Eliecer Gaitán, con los efectos propios de una violencia política y criminal que no ha parado hasta nuestros días. En México es notoria la industrialización de vastas zonas y el consumo de la televisión. Entre tanto, en el cono Sur la preocupación aumenta por el conflicto con las Islas Malvinas.

En medio de las tensiones geopolíticas y una tensa calma que en cualquier momento puede derivar en una guerra catastrófica, se consolida en las regiones del tercer mundo una iniciativa para potencializar la industria y la educación en torno a la ideología tecnócrata. En 1948, por ejemplo, se funda la Universidad Industrial de Santander con un enfoque hacia las ingenierías con programas como Ingeniería Mecánica, Ingeniería Eléctrica e Ingeniería Química ${ }^{11}$. Este es solo un ejemplo entre otras universidades colombianas: Universidad del Valle (1945), Universidad del Atlántico (1946), Universidad de los Andes (1948). Dichas universidades además de compartir el periodo de fundación, comparten la misión, grosso modo, de implementar un desarrollo regional mediante el impulso a industrias pesadas, el apoyo a proceso extractivos de materias primas y, en el mejor de los casos, el desarrollo de innovaciones tecnológicas. De esta forma, se espera contrarrestar los grandes desequilibrios económicos regionales y macro regionales y, sobre todo, entre el norte y el sur. Todo ello bajo el concepto de una apuesta organizada en un marco globalizatorio que consiste en crear polos subregionales de innovación y emprendimiento impulsados por centros especializados de educación superior ${ }^{12}$.

Pero el proyecto va mucho más allá de la construcción de espacios donde se concentre el desarrollo técnico y tecnológico. Tal y como se expone en el apartado anterior, la tecnocracia es consciente de que cualquier forma de conocimiento es vital no solo para el desarrollo científico sino también para el desarrollo social. En razón de ello, inician proyectos para optimizar la educación, al mismo tiempo que se educa a las personas y se induce a una lógica de producción y capitalismo que no permita el afianzamiento de la Internacional Socialista. Es por esta razón que Estados Unidos propone realizar cambios estructurales en la educación y, sobre todo, en los campus universitarios. Dichas reformas son de naturaleza planetaria: en Asia se conoce como Plan Karachi, en África como Plan Addis Abeba y en América Latina llega para el año de 1961 como el Plan Atcon.

Este plan para Latinoamérica es propuesto por el asesor de la Unesco Rudolph Atcon. A grandes rasgos, el modelo Atcon busca agilizar el proceso educativo para

\footnotetext{
${ }^{11}$ Acevedo Tarazona, Álvaro. La UIS: historia de un proyecto técnico cientifico (Bucaramanga: UIS, 1997).

${ }_{12}$ Acevedo, Álvaro. Parque tecnologico Guatiguará: una historia de emprendimiento e innovación tecnológica en Bucaramanga (Bucaramanga: División Publicaciones UIS, 2015), p. 8.
} 
incrementar la producción de mano de obra competente en un oficio. La adecuación educativa implica también crear nuevas técnicas y tecnologías que superen los problemas prácticos y que propendan por el incremento de la rentabilidad de industrias y compañías. Y todo ello, sin desmedro de una formación profesional investigativa y humanística. De la misma manera, busca la reducción de los aportes del estado a la educación y el surgimiento de grandes emporios industriales encargados de la formación de los empleados y el modelamiento de los mismos al gusto del empleador.

Los modelos pretenden estimular la formación humanística pero en la práctica esta formación siempre será subsumida por el afán de alcanzar el progreso humeante y mecanicista de la industrialización. No hay tiempo para detenerse a pensar en la ontología del ser y la máquina, y mucho menos para fomentar programas de humanidades y ciencias sociales. Como el tiempo es extracción y conversión de mercancías, y estas mercancías son dinero, se reestructura y flexibiliza el tiempo de estudio de los aprendices pasando a tres años de formación a la vez que se inauguran los estudios técnicos y tecnológicos que orbitan en un saber hacer. Y ya que se acorta el tiempo del aprendiz en función a la capitalización de los recursos, es necesario realizar currículos que tiendan a la individualización. En otras palabras, hacia un conocimiento fragmentado que no dialogue con otras ramas y enfoques del saber. El propósito es sectorizar a toda costa el conocimiento con fines productivos. El denominado tercer mundo pierde toda esperanza de generar nuevos conocimientos e investigación, pues este saber queda reservado para los institutos ultra científicos y tecnológicos del primer mundo.

Obviamente tales reformas repercuten en la cotidianidad de los estudiantes; se producen malestares y movilizaciones en una juventud hija del desconcierto. Una juventud que parece romper los márgenes culturales y sociales establecidos; una generación de padre obrero o de clase media, educada en un colegio para obreros o clases medias, viviendo en un barrio para obreros o clases medias, alentando los fines de semana el equipo de futbol de los obreros o de las clases medias. Lo que se esperaría, entonces, es un hijo obrero o de clase media de este padre obrero o de clase media. Pero no es así. Este hijo, en algunos casos, deriva en un joven ilusionado con una revolución que conduzca a una sociedad distinta.

El plan reformista de Atcon prevé tales escenarios, por esta razón propone desmovilizar cualquier intento de unificación, estructuración y concertación universitaria para un cambio social por vías de hecho y cercano a la ideología comunista. En su lugar, promueve una reforma educativa funcionalista de tendencia corporativa y pacífica para invadir el organismo social con programas que incidan en el desarrollo de la sociedad. Esta contradicción en América Latina lleva a que en la década de los años sesenta, más que la consagración de bandas de rock, el uso de los anticonceptivos o una aparente revolución cultural en los consumos y formas de ver el mundo de la juventud, se dé inicio a una lucha estudiantil continental con distintas especificidades nacionales y regionales, muy a propósito de los lineamientos globalizados ${ }^{13}$. De aquí

${ }^{13}$ Acevedo, Álvaro. "Ideología revolucionaría y sociabilidad política en los grupos universitarios maoístas 
en adelante, las continuas movilizaciones y protestas de los estudiantes son distintas una tras otra. La razón de esta diferencia radica en que son producciones colectivas, radiografías sociales de una América Latina compuestas por regiones y en un cambio continuo. Pensar en la educación, la región y los problemas globales es entonces un oficio que lleva a evaluar las perspectivas de cada generación que se toma las calles ${ }^{14}$, hace carteles y proclama arengas y utopías en búsqueda de la solución a sus propias angustias y problemas. Esto hace que cada generación sea muy distinta a la anterior y que cada una se refiera a problemas muy específicos de sus regiones y naciones, pero en el fondo tienen el mismo sueño, la misma ontología y las mismas posturas contra discursivas en relación al poder.

\section{La inflexión globalizada del año 1968}

A continuación se analizará la inflexión de las políticas globalizadas y tecnócratas en un escenario puntual: el auge de las movilizaciones y la protesta derivada por las reformas Atcon en la universidad colombiana, visibles en su máxima expresión en el año de 1968 pero con repercusiones hasta el año de 1978. Estas movilizaciones se denominan antisistémicas y tienen como referente las protestas al modelo imperialista norteamericano. La lógica de protesta opta por un acercamiento a ideologías de izquierda y/o comunistas. Los principales escenarios donde se desarrolla la tensión son las universidades, bastiones de la circulación de ideas y la consolidación de un sistema crítico y problematizador de la realidad nacional.

En Colombia el antecedente causal de las movilizaciones de 1968 se da cuatro años antes, con la Marcha del Triunfo del 64; allí estudiantes de la Universidad Industrial de Santander logran su máxima gesta impactando tanto a la opinión pública regional y nacional al caminar a pie unos quinientos kilómetros que separan a Bucaramanga de la capital de Colombia. En una movilización llena de retos con una geografía hostil, los estudiantes expresan su inconformidad hacia el rector Juan Francisco Villarreal; paralelamente inauguran un sentir social compartido al rechazar de manera tajante un modelo de educación universitario norteamericano que se quiere implementar en la UIS sin atender a las condiciones particulares en lo social y económico de la propia región santandereana. Un desconocimiento que puede derivar-como en efecto lo será- en una caricatura del modelo educativo norteamericano ${ }^{15}$.

Esta aparentemente lejanía acontecimental presenta factores trasversales semejantes: todos tienen como catalizador una estructura globalizada por parte de instituciones transnacionales que dictan lineamientos o directrices en lo que se denomina el uso correcto de recomendaciones para los estados, las cuales apuntan directamente a la

de los años 60 y 70 en Colombia”, en Historia Caribe, vol. XI, núm. 28, ene.-jun./2015, pp. 141-178.

${ }^{14}$ El término generación se entiende como el proceso de identidad en la construcción de culturas políticas, y los agravios sociales en la configuración de los movimientos sociales contemporáneos, en Muñoz, Victor. Generaciones: juventud universitaria e izquierdas politicas en Chile y México (Santiago de Chile: Lom, 2011), p. 244.

${ }^{15}$ Acevedo, Álvaro. El fin del comienzo, una época, una marcha, un joven rebelde (Bucaramanga: División Cultural UIS, 2013), p. 44. 
educación como uno de los principales problemas a resolver, en particular, el gasto de dinero público en la educación con índices de tasa de retorno verificables para el sector productivo y la sociedad en general. Por ello, con el paso de los años, se exhortará a países como Colombia y México a seguir el ejemplo de Chile y su doctrina neoliberal. Lo cierto es que en México, partiendo de la realidad chilena, se desaprueban las recomendaciones de la Organización para la Cooperación y el Desarrollo Económico $(\text { Ocde })^{16}$. Esto lleva a un primer punto de inflexión importante a partir de 1968: la aparición en las movilizaciones sociales de demandas locales basándose en una referencia global. Esta tendencia se presentará en todas las movilizaciones a lo largo del siglo XX.

Las movilizaciones protagonizadas por sectores inconformes como los estudiantes, en México, Colombia y Chile presentan una constante de incredulidad frente a los gobiernos de turno, en parte por el incumplimiento de las promesas que estos realizan de manera acelerada a los estudiantes con el fin de levantar huelgas o mitigar movilizaciones en las calles. Esto conduce a una desconfianza en los diálogos para la resolución de conflictos y la participación universitaria. En el caso mexicano esta desconfianza es mayor a la de los otros dos países por la coyuntura que ejerce el Partido Revolucionario Institucional (PRI) como principal decepción política en la organización estudiantil. Si se entra a analizar los motivos que generan las protestas, todo pareciera indicar que los problemas no conocen límites espacio-temporales, pues la educación pública latinoamericana siempre tiene entre sus demandas, una mejora en financiamiento, acceso y permanencia ${ }^{17}$; en el caso colombiano, con el continuo rompimiento de los pactos y acuerdos entre gobierno y estudiantes.

Desde luego existen diferencias que complementan la coyuntura educativa latinoamericana, las cuales están ligadas a decisiones colectivas de índole política. En México las instituciones universitarias son un referente de masificación educativa, también de movilización social y de apertura a clases menos favorecidas. De manera que acontecimientos como el del 68 dejan ver a estudiantes sin ninguna militancia política pero con inquietudes por su futuro. Es así como deciden tomar parte activa en la creación de un sistema educativo nacional que en el cortoplacismo critica al Estado, pero en el mediano plazo lo fortalece. De ahí que las posteriores movilizaciones estudiantiles sinteticen las aspiraciones históricas de la sociedad mexicana para contar con una educación libre, autónoma y de calidad, así esto implique la pérdida de influencia política a lo largo de la historia más reciente y transforme la organización estudiantil en una resistencia frente a la representación institucionalizada y una desconfianza a los partidos políticos ${ }^{18}$.

En Colombia, por otro lado, la violencia política genera un ambiente de tensión que no permite desarrollar un ambiente propicio para la formulación de una reforma educativa que busque la gratuidad y elimine la brecha social en materia educativa. La

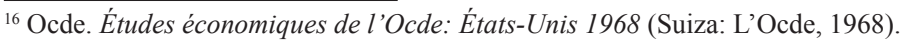

${ }^{17}$ Muñoz, Victor, Op Cit., p. 249.

${ }^{18}$ Ibid., p. 245.
} 
mayoría de los estudiantes que hacen parte de la Marcha del Triunfo del año 64 corren una suerte similar a los casos mexicanos en referencia al incumplimiento continuo de las promesas por parte del gobierno; otros son perseguidos y silenciados, mientras que una minoría termina por el camino del destierro y la lucha armada uniéndose al Ejército de Liberación Nacional (ELN) ${ }^{19}$, el cual tiene su fortín ideológico en los estudiantes de la Universidad Industrial de Santander ${ }^{20}$. El factor violencia potencializado por el miedo y la desaparición lleva a la fragmentación del movimiento estudiantil hasta una eventual invisibilidad en las décadas de los ochenta y noventa. Mientras tanto, se da inicio a una política neoliberal continua con la venta de empresas nacionales y el desentendimiento del papel del Estado en importantes sectores como la salud, la asistencia social y la educación.

En Chile el panorama tiende a distar de las dos realidades anteriores después del golpe de estado al presidente Allende (1973) y la toma del poder por parte de la dictadura; en la educación se acentúan lógicas neoliberales, pero estas no son tomadas como un dictamen de instituciones transnacionales sino como una respuesta pinochetista al déficit político interno. Desde este momento, la educación tiene un fortalecimiento por efecto de la continua inversión privada. Esta inversión sustentada en los principios más básicos del lucro genera una serie de aranceles y sistemas de crédito que convierten a la educación superior chilena en un mecanismo de filtraciones sociales que fortalece a la élite y multiplica nuevas universidades privadas. En este orden de comparaciones, la organización estudiantil chilena es distinta a la de los casos mexicano y colombiano. La Federación de Estudiantes de Chile tiene una dinámica de congresos constituyentes convocados por los centros de alumnos en los que se crean estatutos y hay elecciones universales de la federación. Esta tendencia demuestra una marcada traza institucionalista tanto en las formas como en los mecanismos de la organización estudiantil21 ${ }^{21}$ De manera que el activismo estudiantil de los líderes y cuadros políticos busca afianzar una carrera política o empresarial en su porvenir.

Las continuas apariciones de los estudiantes en las decisiones políticas generan en los países distintas formas de asumir la movilización estudiantil. Tanto por incredulidad en la política como por temor a la violencia o la aceptación de una neoliberalización galopante por un sistema político especializado en la desaparición y la persecución institucionalizada, los estudiantes y sus luchas generacionales son un ejemplo de resistencia. Esta posición de lucha es un sentimiento derivado de un instinto por sobrevivir, lo que faculta la resistencia y la consecución de algunas mejoras en lo que respecta a la transformación regional y la lucha por la educación.

\footnotetext{
${ }^{19}$ Acevedo, Álvaro, El fin del comienzo..., p. 45.

${ }^{20}$ Acevedo, Álvaro. La experiencia histórica del cogobierno en la Universidad Industrial de Santander: concepciones y divergencias en disputa por la autonomía universitaria, 1971-1976 (Bucaramanga: UIS, 2016); Acevedo, Álvaro. Memorias de una época: El movimiento estudiantil en Colombia en los años sesenta y setenta del siglo XX (Bucaramanga: Universidad Industrial de Santander, 2016).

${ }^{21}$ Muñoz, Víctor, Op Cit., p. 245.
} 


\section{La inflexión globalizada del año 2011}

Con el afianzamiento de las lógicas neoliberales en gran parte del mundo, la universidad entra en un proceso de adaptación frente a estándares internacionales de calidad. Similar a la inflexión del año 1968 que tiene su dinamizador cuatro años antes (la marcha del 64), la inflexión del 2011 inicia tres años antes con la crisis económica global que pone en tela de juicio las cualidades innovadoras y dinamizadoras del neoliberalismo. La crisis planetaria tiene su origen en el alza repentina de las materias primas efecto de la especulación y una burbuja crediticia con la cual se pagan hipotecas, créditos de libre inversión y matriculas educativas en todo el mundo. La crisis económica se traduce como la situación en la cual las personas no pueden pagar lo que consumieron, consumen y planean consumir. Esta situación despierta malestares generalizados en la población de todo el mundo; tan solo en Latinoamérica grandes movilizaciones como Yo soy 132, la Mane, etc., son conocidos de manera general como los indignados. Si bien los distintos grupos hacen énfasis a necesidades puntuales, tienen en común algunos apartes del discurso y ciertas estrategias con las cuales convocan fenómenos colectivos para hacer las reclamaciones a los gobiernos.

Yo Soy 132 en México responde a las necesidades de una generación de nativos digitales que buscan una democratización de los medios de comunicación y un rechazo a la manipulación por parte de grandes consorcios informativos e interactivos mexicanos. La dinámica de estos nuevos colectivos sociales no representa un gremio o grupo humano; la fuerza proviene de la heterogeneidad de los individuos que conforman el colectivo al igual que en los múltiples campos de acción que van desde una lucha por los derechos digitales, pasando por una conciencia ecológica hasta los más agudos problemas nacionales como la corrupción, la desigualdad social, los ajustes educativos y la necesidad esclarecer la fuerza desmedida del estado en los disturbios de Atenco en el año 2006. La consigna de \#YoSoy132 apunta hacia la inclusión y la pregunta por una nación mexicana que atienda el creciente malestar social. Lo que parece ser cuestión fugaz del momento, crece de manera considerable y hace tambalear la popularidad del presidente Peña Nieto. Más que una respuesta propositiva que logre un cambio radical, Yo Soy 132 permite que ciudadanos mexicanos se conozcan entre sí y se organicen para la protesta ante problemáticas educativas, energéticas y culturales.

Por su parte, la Mesa Amplia Nacional de Estudiantes (Mane) en Colombia, surge del descontento por los continuos recortes a la educación superior y la intención del gobierno colombiano de emular el modelo educativo chileno que se fundamenta en la educación con ánimo de lucro. La población que se manifiesta también es joven, en su mayoría universitarios, y expresa una preocupación por el proyecto de reforma a la Ley 30 de 1992. Al constituirse Colombia como un país donde la violencia es un fenómeno trasversal de su cotidianidad, los manifestantes rompen cánones anteriores de la protesta violenta y hacen movilizaciones no violentas, en las que apelan a la recursividad por medio de marchas-carnavales, desnudatones, besatones y múltiples acciones colectivas que capturan la atención de la comunidad en general y de los medios de comunicación. Además, crean sus propios canales de comunicación 
independientes usando plataformas como YouTube, los estudiantes hacen videos mostrando la realidad de la educación. El centro de la discusión es hacer una reforma, pero para mejorar y no para vender al mejor postor cualquier educación tecnócrata y sin investigación. Al final el gobierno se ve obligado, por la presión pública, a archivar la propuesta de reforma educativa y a enviar tanto a su gabinete como a los distintos actores educativos -sugiriendo incluir a la Mane- a reestructurar una propuesta concertada. Lo que a la postre no se alcanzará. De igual manera, los manifestantes están al tanto y apoyan las protestas campesinas que luchan por los inflados precios de fertilizantes, combustibles y por la obligación de comprar semillas transgénicas. También hacen presencia ante la incursión de proyectos de mega minería en regiones como el páramo de Santurbán.

Por su parte, en Chile se intensifican las movilizaciones de los estudiantes en general, con un rechazo rotundo a un sistema de educación en el cual el estado solo apoya con un $15 \%$ y $25 \%$ de inversión, mientras que los mismos estudiantes son quienes tienen que sufragar el restante por medio de créditos educativos que hipotecan su futuro en los próximos quince o veinte años. Entre los manifestantes se encuentra la Federación de Estudiantes de Chile y la Federación de Estudiantes de la Universidad de Chile. Las manifestaciones en Chile son bastante controladas por la fuerza pública, sin descontar que los manifestantes son instigados por los carabineros. Para evitar el uso de la fuerza y la violencia por ambas partes, los estudiantes idean nuevas formas de movilización, como es el baile masivo de Thriller el 23 de junio frente al Palacio de la Moneda: una representación de la zombificación en la que se encuentra la educación pública chilena ${ }^{22}$. De la misma manera, muestran su preocupación por medio de medios virtuales creando portales web independientes como YoDebo.cl, en el que jóvenes chilenos dan a conocer las escandalosas sumas de dinero que adeudan por créditos estudiantiles. Con la elección de la presidenta Bachelet se cree que las cosas van a mejorar. Se espera darle acceso educativo gratuito a un $50 \%$ de los jóvenes más pobres de Chile. Si bien falta mucho para mejorar el sistema educativo chileno, en el año 2011 la presión es suficiente para que las cosas empiecen a cambiar.

En los tres casos anteriores existen similitudes. En primer lugar, se encuentra la participación de individuos autónomos en movilizaciones, porque cada quien siente que está afectado en el futuro de sí-mismo. En segundo lugar, no hay una masa, un gremio o un grupo social único. Esto se evidencia en que los jóvenes que se suman a las protestas de hoy no creen en metarrelatos ni en aspiraciones maximalistas, como sí creyeron los jóvenes del 68. Metarrelatos y aspiraciones que, en su modo de ver, son construidos únicamente para que los políticos ganen adeptos y puedan conservar sus intereses de poder. En tercer lugar, hay una renovación en las estrategias de protesta. Los jóvenes de hoy no necesitan tomar las armas como único medio para hacer sentir sus reclamos; prefieren apelar a formas de protesta amenas, divertidas y que se alejen de cualquier intento de violencia contra la fuerza pública. En cuarto lugar, no hay que pensar que estos jóvenes son contraculturales como los jóvenes de los años sesenta.

\footnotetext{
${ }^{22}$ Figueroa, Francisco. Llegamos para quedarnos. Crónica de la revuelta estudiantil (Santiago de Chile: LOM, 2012), p 95.
} 
De hecho, son todo lo contrario, son omnívoros culturales que implementan todas las formas ficticias consumidas por los medios de comunicación (como los zombis, goku, etc.) para mostrar su desacuerdo. En otras palabras, los jóvenes latinoamericanos de hoy usan los elementos de la globalización en contra del propio sistema y de esta manera generan una riqueza conceptual en las movilizaciones e indignaciones.

Existe un elemento particular que debe ser analizado en cualquier intento por desarrollar historia regional o local en estudios de la educación, y es que es por medio de la misma globalización, cuya finalidad es superponer las barreras espacio-temporales para de esta manera compartir múltiples realidades ${ }^{23}$, los estudiantes pueden compartir estas realidades y llevar a cabo movilizaciones latinoamericanas. Es el caso de la Marcha Latinoamericana por la Educación realizada el 24 de noviembre de 2011, la cual reúne estudiantes de todas las regiones del continente para compartir la necesidad de luchar por una educación mejor que no empeñe su futuro y que, en lugar de penalizar a quien quiere estudiar e investigar, aliente los procesos educativos que lleven a pensar la región, a pensar Latinoamérica, y a creer en un cambio en el que exista un porvenir que no sea utópico sino construido basándose en la alteridad y el conocimiento. Y es en estas condiciones que surge la esperanza. Marc Augé al ser abordado e interrogado por temas como el terrorismo, los fanatismos religiosos, la corrupción, la desigualdad social y la crisis ecológica que amenazan al planeta entero, cree que la esperanza está en la educación ${ }^{24}$.

\section{A modo de cierre. La rebelión de los esquizos: ¿continuidad en las inflexiones?}

Es habitual que ciertos analistas consideren las protestas del 68 como un macroacontecimiento que tiene efectos poderosos con las protestas de hoy. Quienes alimentan esta idea ignoran el pragmatismo de las movilizaciones contemporáneas. Si los jóvenes de hace medio siglo son utópicos porque tienen en su mirada la esperanza del cambio y son optimistas porque todo puede ser mejor, los jóvenes de hoy que se toman las calles, en cambio, son pesimistas, y este pesimismo, más que ser considerado una cualidad, es tachado de falta de esperanza. No obstante, sobre este pesimismo dijo el Nobel de Literatura, José Saramago, adelantándose a los indignados y el movimiento global: “[...] los únicos interesados en cambiar el mundo son los pesimistas, porque los optimistas están encantados con lo que hay" 25 . El pesimismo es síntoma de un problema que consume al mundo y este va de la mano con problemas económicos, geopolíticos, de fanatismos religiosos, ecológicos y, sobre todo, históricos y filosóficos.

En su momento, el psicoanalista Lacan enfrenta a los jóvenes parisinos que se toman las calles en mayo de 1968: “[...] a lo que ustedes aspiran como revolucionarios es a un

\footnotetext{
${ }^{23}$ Fazio, Hugo. ¿Qué es la globalización? (Bogotá: Uniandes, 2011).

${ }^{24}$ Navarrete, Steven. "La educación es nuestra única esperanza: Marc Augé”, El Espectador, 5 de noviembre de 2015 .

${ }^{25}$ Entrevista a José Saramago, Bogotá, 28 de noviembre de 2004.
} 
amo $[\ldots]$ no se hagan ilusiones, muchachos, ustedes no serán jamás sus propios amos, lo único que lograrán será cambiar sus cadenas" ${ }^{\text {"26 }}$. No se sabe a qué se refiere Lacan con las cadenas, pero es posible que sean las cadenas del consumo de los estudiantes de hoy, las cuales aceptan consumiendo más mientras aumentan su capital cultural. Y aquí está la paradoja: en 1968 surge un cuestionamiento a la familia, los medios y la educación. Esto implicará protestar para buscar el cambio. Sin embargo, el cambio no llega. Menos llega la liberación ni la justicia ni la igualdad para todos. Y tampoco se logra la crítica a toda forma de autoritarismo, pues son los mismos estudiantes que protestan contra el autoritarismo los que se muestran autoritarios con sus credos y ortodoxias ideológicas ${ }^{27}$.

El capitalismo muta permanentemente para presentar una nueva cara. Los conceptos que definen el capitalismo industrial son antagónicos frente al nuevo capitalismo de consumo que desaloja cualquier forma de radicalización. El capitalismo de consumo de hoy promueve interrelaciones que buscan evitar conflictos, desencuentros, debates y confrontaciones de opiniones. Señala que comparte conceptos por una sociedad ecuánime en la que nadie sea discriminado por ser distinto, pero, eso sí, siempre y cuando la diferencia no discuta con el amplio espectro de consumo producido por este mismo capitalismo. Esta es la esencia de la hipermodernidad ${ }^{28}$.

El capitalismo de consumo es un sistema enfermo, tiene esquizofrenia. Y, sin embargo, hoy ya no es un capitalismo salvaje. Ha mutado a un capitalismo amable que busca el respeto por el otro y consagra luchas individualizadas de género, ecológicas y de respeto por las minorías ${ }^{29}$. La explicación ontológica a esta nueva tendencia enfrenta al ser a una incertidumbre frente al sistema, pues este no sabe diferenciar si él es el problema o es el capitalismo que encarna luchas altruistas en defensa por la humanidad. Y, sin embargo, este ser no encaja en el sistema, cayendo de esta manera en la desesperanza. Por eso es que hoy ya no existen revolucionarios sino depresivos ${ }^{30}$. Los hijos del capitalismo de consumo no creen en las utopías sesenteras: la falta de entusiasmo es lo que caracteriza la actualidad. Hay pocas cosas que los hacen soñar; las ideologías no lo hacen. Las utopías se han centrado en metas pequeñas, ya no se piensa en cambiar el mundo ${ }^{31}$. La educación y las movilizaciones latinoamericanas, e incluso las mundiales, ya no apelan a una ideología política sino a pulsiones básicas, a necesidades mitológicas de supervivencia como el porvenir en un futuro mejor o la esperanza de un mejor futuro; también recurren a la educación como un derecho fundamental, el cual no conoce latitudes, contextos, historias y contornos sociales.

\footnotetext{
${ }^{26}$ Castro-Gómez, Santiago. Revoluciones sin sujeto: Slavoj Zizek y la critica del historicismo posmoderno, (Bogotá: Akal, 2015), p. 13.

${ }^{27}$ Feria-Cuevas, Yolanda. “1968 año de rebeliones”, en Educ@arnos, revistaeducarnos.com/2015/10/01/1968ano-de-rebeliones/ (10 de noviembre de 2015).

${ }^{28}$ Lipovetsky, Gilles, Op Cit., p. 328.

${ }^{29}$ Castro-Gómez, Op Cit.., p. 15.

${ }^{30}$ Chul Han, Byung. Psicopolitica: neoliberalismo y nuevas técnicas de poder (Barcelona: Herder, 2014), p. 18.

${ }^{31}$ Navarrete, Steven. "El capitalismo es una máquina de seducción", El Espectador, 5 de septiembre de 2015 .
} 
Las nuevas formas de protesta levantan todo tipo de opiniones tanto en quienes están a favor como en quienes están en contra. Empezando porque estas no representan una ideología dominante y totalizadora. Los jóvenes de hoy no se autodefinen a sí mismos en sus protestas como un movimiento antisistémico. Ellos reconocen que están inmersos en una sociedad que capitaliza absolutamente todos los recursos del planeta para después capitalizar a los actores sociales y, por último, hacer lo mismo con lo que está aconteciendo: el futuro.

En esta nueva instancia, mejor conocida como la segunda modernidad, hay un proceso continuo por capitalizar el sí-mismo, y la mejor forma de invertir es por medio de la educación. Por tal razón aparecen los cursos de idiomas, en especial de inglés. También los cursos rápidos con el afianzamiento de la web 2.0 y las tecnologías de la información y las comunicaciones. Así mismo, los cursos virtuales diseñados por las universidades para tener un rango de acción superior y generar recursos sin la inversión en infraestructura propicia para la investigación, optimizando el margen de estudiantes por docentes e incrementando el número de cursos mientras se interioriza cada vez más una educación sin ningún contacto interrelacionado, pero sí emulando una comunicación virtual que no es más que un intercambio binario de códigos entre dos avatares que muy dudosamente representan lo que se es en la materialidad, pero, de seguro, recoge las añoranzas del actor social en un medio en el que las posibilidades solo son limitadas por el ancho de banda.

Las derivaciones continuas de la tecnocracia y la globalización alteran el panorama latinoamericano generando movilizaciones sociales de las cuales los jóvenes son los principales protagonistas. Es casi una obligación de los investigadores sociales con la sociedad y su tiempo, generar nuevos horizontes interpretativos desde los cuales sea posible abordar una historia glocal; una historia que respete las conexiones que existen en una era social de la comunicación, pero también que brinde un horizonte interpretativo y que permita dilucidar el ritmo y la ruta que toma la región, pues esta no debe quedar a merced únicamente de la planeación y de los intereses geopolíticos. Las nuevas formas de activismo, a las que se suma el ciberactivismo, demuestran una preocupación continua de los actores sociales por las problemáticas políticas de su localidad: localidades unidas una tras otra en una mega-red local. El historiador debe comprender tales derivaciones y establecer análisis que plasmen, en su interrelación, a este sujeto; un sujeto que se niega a ser atomizado, y que rechaza verse a sí mismo como un gadget o un mecanismo en la máquina devoradora de almas que es la sociedad masificada. El actor se encuentra en un enjambre, un enjambre local, y solo desde su deseo, sus intenciones y autonomía posiblemente quiere hacer parte del cambio, un cambio en el que necesita del otro. 
La universidad latinoamericana frente a la globalización. Continuidades e inflexiones...

\section{Fuentes}

\section{Fuentes primaria}

\section{Publicaciones periódicas}

El Espectador, Bogotá, 2004.

\section{Libros}

Ocde. Études économiques de l'Ocde: États-Unis 1968. Suiza: L’Ocde, 1968

\section{Publicaciones en Internet}

Feria-Cuevas, Yolanda. 1968 año de rebeliones, www.revistaeducarnos. com/2015/10/01/1968-ano-de-rebeliones/.

Real Academia de la Lengua Española. 2015. http://dle.rae.es/?id=0FhRgFA.

\section{Fuente secundaria}

\section{Libros}

Acevedo, Álvaro. El fin del comienzo, una época, una marcha, un joven rebelde. Bucaramanga: División Cultural UIS, 2013

Acevedo, Álvaro. Parque tecnológico Guatiguará: una historia de emprendimiento e innovación tecnológica en Bucaramanga. Bucaramanga: División Publicaciones UIS, 2015

Acevedo, Álvaro. La UIS: historia de un proyecto técnico científico. Bucaramanga: UIS, 1999.

Acevedo, Álvaro. La experiencia histórica del cogobierno en la Universidad Industrial de Santander: concepciones y divergencias en disputa por la autonomía universitaria, 1971-1976. Bucaramanga: UIS, 2016. 230 p.

Acevedo, Álvaro. Memorias de una época: El movimiento estudiantil en Colombia en los años sesenta y setenta del siglo XX. Bucaramanga: Universidad Industrial de Santander, 2016.

Amis, Martin. Los monstruos de Einstein. Barcelona: Minotauro, 1990

Barraclough, Geoffrey. Introducción a la historia contemporanea. Madrid: Gredos, 1964 
Chul Han, Byung. Psicopolitica: neoliberalismo y nuevas técnicas de poder. Barcelona: Herder, 2014.

Fazio, Hugo. ¿Qué es la globalización? Bogotá: Uniandes, 2011

Figueroa, Francisco. Llegamos para quedarnos. Crónica de la revuelta estudiantil. Santiago de Chile: LOM, 2012

George, Susan. Otro mundo es posible si... Barcelona: Icaria, 2004.

Lipovetsky, Gilles. La estetización del mundo: vivir en la época del capitalismo artístico. Barcelona: Anagrama, 2015.

Marcuse, Herbert. Contrarrevolución y revuelta. México D.F: Ed. Joaquín Mortiz, 1973.

Muñoz, Victor. Generaciones: juventud universitaria e izquierdas politicas en Chile y México. Santiago de Chile: Lom, 2011.

Robertson, Roland. Glocalización: tiempo-espacio y homogeneidad-heterogeneidad. Madrid: Trotta, 2003

Touraine, Alain. La sociedad postindustrial. Barcelona: Ariel, 1973

Wallerstein, Immanuel. El moderno sistema mundial. Buenos Aires: Siglo XXI, 1979

Walter, Malcolm. Globalization. Londres: Routledge, 1995

Zizek, Slavoj. Acontecimiento. Madrid: Sexto Piso, 2014.

\section{Artículos en revistas}

Acevedo, Álvaro. "Ideología revolucionaría y sociabilidad política en los grupos universitarios maoístas de los años 60 y 70 en Colombia", en Historia Caribe, vol. XI, núm. 28, ene.-jun./2015, pp. 141-178.

Mayol, Alberto. "La tecnocracia: el falso profeta de la modernidad", en Revista de Sociología, núm. 17, Santiago de Chile: Universidad de Chile, 2003, pp. 95-123. 